\title{
COMPETENCIAS DEL DOCENTE \\ UNIVERSITARIO. \\ DESAFÍOS PARA LAS UNIVERSIDADES \\ PRIVADAS DE EL SALVADOR
}

Aydeé Rivera de Parada ${ }^{1}$ Directora de Investigaciones Universidad Evangélica de El Salvador aydee.parada@uees.edu.sv

\author{
Los docentes han dejado de ser parte de la proporción más formada \\ de la población en los distintos países. En la medida en que la educación \\ secundaria y superior se extienden, tener título docente ya no califica a quien \\ lo posee de manera sustantivamente diferente de los otros miembros de la \\ propia generación, a diferencia de lo que sucedía un siglo atrás... \\ Flavia Terigi, 2010 \\ Recibido 15/02/16 \\ Aprobado 20/05/16
}

\section{Resumen}

Este artículo tiene el objetivo de identificar competencias y elementos académicos del docente universitario que contribuyen a la calidad de enseñanza en educación superior, para proponer estrategias de formación continua del docente en las universidades privadas, mostrando el dominio de elementos que definen las competencias genéricas de la docencia superior. El estudio es de carácter descriptivo, con análisis predominantemente cuantitativo, participaron 384 docentes de 22 universidades privadas de El Salvador, contactados individualmente en las universidades y por medio de jefaturas académicas. Los resultados muestran docentes con conocimientos, disposición e interés por contribuir a la educación, que reconocen las competencias docentes como base de la calidad universitaria; pero con capacidad mediana de dominio y apropiación de elementos esenciales. La concepción de calidad que subyace en la representación mental de docentes también pasa por los elementos organizacionales y de infraestructura alrededor de la formación de los estudiantes, también incluye la responsabilidad de la institución para promover la estimulación económica, la educación y capacitación continua de la planta docente. Se recomienda estrategias de actualización inter universitarias y la incorporación de indicadores que ayuden a monitorear la evolución de la calidad docente.

PALABRAS CLAVE: Docente universitario; indicadores de competencia docente; calidad docente; formación continua; universidades privadas; El Salvador.

1 Educadora, en proceso de graduación del Doctorado en Ciencias Sociales por la Universidad del Zulia de Venezuela. 


\section{FACULTY COMPETENCIES: CHALLENGES FOR PRIVATE UNIVERSITIES IN EL SALVADOR}

\section{Summary}

This article aims to identify skills and academic elements of teaching university, which supply to the eminence of teaching in higher education, to propose the training strategies of teachers in private universities, showing the field elements that define the generic competencies of higher teaching. The study is descriptive, with a predominantly quantitative analysis, 384 teachers of 22 private universities in El Salvador participated, in which they were individually contacted through universities and academic headquarters. The results show teachers with knowledge, willingness and interest to contribute to education, which recognize teaching skills as the basis of university worth; also, with medium capability domain and ownership of vital elements. The concept of quality that underlies the mental depiction of teachers goes through organizational elements and infrastructure around the training of students; it also includes the responsibility the institution, the promotion of economic stimulation, education and continuous training of the faculty. Inter-university strategies are recommended to be continuously updated and to integrate the indicators to help observe the evolution of teaching quality.

KEYWORDS: University teacher; teaching competence indicators; teaching quality; continuous training; El Salvador.

\section{INTRODUCCIÓN}

En El Salvador existen 24 universidades, entre ellas una es estatal, nueve institutos especializados y siete institutos tecnológicos. Según el Ministerio de Educación (MINED, 2015), para el periodo 2010-2014, las universidades reportaron en su planta docente un incremento aproximado de 500 profesionales, la mayoría con tipo de contratación hora clase; entre ellos prevalece el grado universitario, la cuarta parte con nivel de maestría y solo dos de cada cien tienen doctorado de posgrado. Se observa tendencia de aumento en la formación de masters, pero siguen siendo escasos los docentes con doctorado de posgrado; se desarrollan pocos programas sistemáticos de actualización docente; mientras en la mayoría de universidades se pretende formar profesionales por competencias, la formación continua de la docencia universitaria tiende a mantenerse en un modelo inductivo teórico.

Muchos de los docentes universitarios llegaron a las aulas sin tener preparación especializada en docencia, porque se les dio la oportunidad, sin embargo es importante reconocer que buena parte de ellos se han interesado en formarse en el ejercicio, aunque no todos alcanzan el nivel de competencia requerido para lograr la verdadera acción de "enseñar a aprender", de motivar el pensamiento crítico, desarrollo de ciudadanía en los estudiantes, y mucho menos la producción científica. 
Diaz Barriga (2005) y Salinas (2004) aseveran que, hasta los años setenta del siglo XX, al profesor universitario solo se le exigía tener dominio de los contenidos de la disciplina objeto de su enseñanza, pero en la actualidad debe incorporar técnicas y estrategias didácticas, así como el uso intensivo de tecnologías de información y comunicación, así mismo el aprendizaje de otros idiomas como el inglés y ejes transversales de enseñanza según las necesidades del contexto.

El factor de formación docente, la vocación, así como elementos de formación científica y cívica, son determinantes para la labor efectiva y ser congruentes con la formación profesional de verdaderos ciudadanos comprometidos con el entorno social.

Tanto las conferencias de educación superior como otros acuerdos internacionales hacen énfasis en el compromiso y disposición de las autoridades universitarias para dar a la educación superior el lugar que debe tener en la sociedad, como generadores de conocimiento y entes propositivos de soluciones a los problemas sociales; de igual manera, en el proyecto Tuning América Latina, se refuerza el enfoque de pertinencia socioeconómica. Estas propuestas generan el compromiso de las universidades en ser congruentes con el mercado global y la dinámica social del mundo. Sin embargo, el presente estudio suma la reactivación del componente metodológico y didáctico para desarrollar la base formativa de docentes universitarios con fuerte compromiso social y colaboración inter institucional.

La excelencia del profesor siempre es el resultado de largos procesos de formación, donde no basta la pasión y voluntad, sino se agrega la probidad académica, que solo se logra con asertiva formación y experiencia.
En este sentido, el docente de cualquier nivel debería resultar por vocación, y especialmente el docente universitario debería tener no solo el interés para crear conocimiento nuevo y enfocarse al desarrollo de la sociedad, sino también las capacidades para que sus aportes académicos y científicos, dentro y fuera del aula, sean de alto impacto, con profunda pasión por lo que hace posible el crecimiento intelectual $\mathrm{y}$ humano de los estudiantes, "el docente universitario debe ser alguien que sienta una extraña embriaguez por lo académico", Weber (1979).

La necesidad de conocer mejor a los docentes de educación superior lleva a plantear las siguientes interrogantes: ¿Cuáles son las competencias y elementos académicos del docente que contribuyen a la calidad de enseñanza en educación superior? ¿Cuáles son las estrategias de formación del docente universitario que podrían propiciar la actualización permanente en las universidades privadas?

\section{ANTECEDENTES}

Un problema recurrente en la universidad es encontrar docentes que son profesionales que conocen su disciplina, pero que no necesariamente aprendieron cómo enseñar, aunque para un gran número de profesores en estas situaciones, este hecho no tiene mayores implicaciones en el contexto educativo (Tovar-Gálvez, 2012). Diferentes estudios han concluido que la enseñanza de una disciplina específica se sustenta en el dominio de un conjunto de conocimientos pedagógicos, curriculares, de los estudiantes, del contexto y del contenido que se enseña, entre otros.

Otro aspecto clave es el dominio de la lectura y escritura. Garrido (2014) se 
refiere a resultados de la lectura y escritura de estudiantes de primer ingreso: en una evaluación de 1 a 10, alcanzan 5.9 en comprensión de la lectura, lo que debe ser preocupante para las IES mexicanas y las instituciones de formación básica y, aún más allá, a la formación y al desempeño de los maestros del preescolar al posgrado.

La lectura posibilita la capacidad de entender, dar y pedir explicaciones, la continuidad del aprendizaje, permite la multiplicación de experiencias, favorece la capacidad de ser autodidactas (cada quien sabe hasta dónde lee), es una manera de adiestrarse en el arte de pensar. $\mathrm{Su}$ rendimiento es consecuencia de su calidad de lectores capaces de escribir, esos mejores alumnos serán mejores trabajadores, mejores profesionales, empresarios y políticos más capaces y podrán armar sociedades más prósperas y justas, donde se disfrute de un nivel de vida más alto.

Lo anterior le exige al docente también ser lector capaz de comprender para escribir, pues mientras no se logre hacer lectores a los maestros y convertirlos en los más importantes promotores de la lectura y la escritura, jamás habrá una población mayoritariamente lectora. Leer y escribir son dos condiciones obligatorias para los académicos, es la base para el desarrollo de competencias porque mejora las estrategias de aprendizaje.

A partir de los modelos pedagógicos, es necesario organizar la infraestructura tanto de recursos como de capacitación del profesorado. "Cambiar las estructuras de aprendizaje, no se logra con el desarrollo de unos contenidos que enuncien cómo hacerlo; la transformación debe ser parte de la práctica pedagógica, lo cual implica que los profesores deben estar comprometidos" para lograr los propósitos que llevan al cambio de paradigmas de enseñanza de los profesores y de aprendizaje por parte de los estudiantes (León y Risco, 2014).

Álvarez (2011) presenta una aproximación del perfil del docente basado en competencias en una universidad de Argentina, partiendo de la necesidad que el estudiante y el docente hagan cambios significativos para logar el aprendizaje interactivo con la realidad, con base en conocimientos previos de su contexto social y profesional; de manera que el estudiante debe cambiar el aprendizaje memorístico por la comprensión más holística de la vida con actitud autodidacta, mientras el docente debe disminuir las disertaciones y adoptar nuevas funciones. Seguidamente, Álvarez presenta un perfil resumido de la siguiente manera: tener un conocimiento en lo disciplinar y pedagógico, tener habilidades de gestión y planificación eficiente de la enseñanza, utilización de recursos de acuerdo a diferentes contextos, capacidad de tutoría, orientar el auto aprendizaje individual y colectivo, incentivar al estudiante, tiene capacidades culturales y contextuales, las capacidades comunicativas, sociales, cognitivas, meta cognitivas entre otras.

\section{ENSEÑAR POR COMPETENCIAS}

Las competencias son "complejas capacidades integradas, en diversos grados, que la educación debe formar en los individuos para que puedan desempeñarse como sujetos responsables en diferentes situaciones de la vida social y personal, sabiendo ver, hacer, actuar y disfrutar convenientemente, evaluando alternativas, eligiendo las estrategias adecuadas y haciéndose cargo de las decisiones tomadas" (Cullen, 1996, citado por Beneitone 2007). Según esta definición, no se trata solo de ser efectivos 
en el campo laboral, las competencias son un conjunto de saberes, actitudes, habilidades y responsabilidades que se deben desarrollar en los estudiantes para hacer sujetos asertivos en la vida. Esto demanda un alto grado de desempeño en los docentes que tienen a cargo dicha formación.

Jacques Delors (1996), en el Informe de la Comisión Internacional sobre la Educación para el Siglo XXI, proponía una visión integrada de la educación. En continuidad con el Informe Faure (1972), en donde incorpora el aprender a ser, el Informe Delors se considera una referencia clave para la conceptualización de la educación y del aprendizaje en todo el mundo, en donde incorpora los paradigmas del aprendizaje a lo largo de toda la vida y los cuatro pilares del aprendizaje: aprender a conocer, aprender a hacer, aprender a ser y aprender a vivir juntos. Considera las tensiones existentes entre lo local y lo mundial, en el plano personal, la salud física y psicológica, lo material y lo espiritual, la necesidad de hacer evidente la comprensión del otro y convivir en armonía; y que estas tensiones deben estar contempladas en la planeación educativa.

El proyecto Tuning para América Latina (2007), como adaptación del Tuning europeo, es una fuente de inspiración en la mejora curricular, pues aporta interesantes reflexiones para repensar las estrategias didácticas en educación superior. La metodología Tuning incluye el desarrollo de competencias genéricas y específicas como primera línea. De manera que las competencias genéricas implican la convivencia y valores de una persona integral, de comunicación y autoformación, desarrollo de pensamiento crítico y razonamiento lógico con base en el conocimiento de la realidad, mientras que las competencias específicas son las necesarias en cada disciplina para ser técnico o experto en un área especializada. Plantea la concepción de competencias en un sentido integral, "que conducen a una persona responsable a ser competente para realizar múltiples acciones (sociales, cognitivas, culturales, afectivas, laborales, productivas) por las cuales proyecta $\mathrm{y}$ evidencia su capacidad de resolver un problema dado, dentro de un contexto específico y cambiante" (Beneitone, Esquentini y col., 2007).

Los mercados laborales son más dinámicos y flexibles que los mercados educativos. La rigidez y obsolescencia de los programas educativos aleja cada vez más la posibilidad de que los egresados encajen completamente en el mundo laboral (Rama, 2016). Paradójicamente, no son las competencias específicas per se las que van a lograr hacer eficiente a un profesional, porque el conocimiento se afecta con las innovaciones en cada ámbito laboral, y esto implica haber aprendido a aprender.

Otro factor clave en la formación académica es la cultura política y cultura cívica para la convivencia ciudadana responsable (no como ideología partidarista), en tanto representa la base política de las sociedades; sin embargo es poco cultivada, debido a la priorización de la instrucción profesional en las universidades privadas.

La "ciudadanía" se debe entender como las capacidades cognitivas, morales, organizativas, relación emocional y afectiva de su entorno social. En el ámbito universitario, el desarrollo de una cultura política y cívica es una labor que los docentes pueden y deben hacer. Para fortalecer el desarrollo de verdaderos ciudadanos, no solo es necesario desarrollar conocimientos, sino inducir al sentido crítico y cambio 
de actitudes consientes de participación asertiva. En las universidades hay una aproximación escasa al desarrollo de una cultura política democrática, aunque la educación en El Salvador, según la Ley General de Educación, tiene entre sus fines esenciales "Lograr el desarrollo integral de la personalidad en su dimensión espiritual, moral y social" (MINED, 2005).

\section{METODOLOGÍA}

Se realizó un estudio de nivel descriptivo, con predominio cuantitativo, del que se deriva este artículo. Participaron 384 docentes de 22 universidades privadas, por medio de una muestra probabilística estratificada. Una parte del cuestionario general ubica la visión que tiene el docente sobre sus propios conocimientos y dominios para contribuir a la calidad universitaria.

\section{ANÁLISIS Y DISCUSIÓN DE RESULTADOS}

De los docentes participantes, el 54.9\% corresponde a hombres y el $44.3 \%$ corresponde a mujeres. Por el tipo de contratación, son docentes tiempo completo $64 \%$, hora clase $30 \%$ y tiempo parcial 5.0\%. El 76.6\% tienen formación en docencia. El 52\% tienen grado universitario, docentes con maestría 41\% y de doctores de posgrado 6\%, a diferencia del dato de país, que muestra que los docentes contrastados con maestría es de $24 \%$ y doctores de posgrado en $2 \%$.

Según los mismos docentes, el elemento más importante para definir la calidad universitaria son las competencias docentes con 45.3\%, seguido de la organización institucional con 23.4\%, y recursos disponibles con $18.2 \%$. Coinciden con López (2012) cuando afirman que el docente es quien marca el nivel de calidad que puede alcanzar la IES, y con Weber (1979), quien reconoce que el docente debe sentir una extraña embriaguez por lo académico, es decir, mucha identificación con la universidad, por prepararse $\mathrm{y}$ actualizarse constantemente.

Al menos el $80 \%$ de los docentes califican como excelente y muy bueno a los recursos y condiciones que la universidad les brinda para su desempeño.

Los docentes consideran que todos los elementos planteados como parte del proceso de enseñanza y de aprendizaje son muy importantes; todos los puntajes son mayores al $80 \%$ en la categoría de mucha importancia, excepto el conocimiento y promoción de cultura política, que aparece un poco más bajo con $70 \%$. Ver tabla 1 .

Tabla 1. Qué tan importantes son los siguientes elementos (competencias y habilidades) en la formación de estudiantes

\begin{tabular}{|c|c|c|c|c|c|c|c|c|}
\hline $\begin{array}{c}\text { Nivel de } \\
\text { importancia/\% }\end{array}$ & $\begin{array}{l}\text { 1. Conocer } \\
\text { y promover } \\
\text { cultura } \\
\text { política } \\
\text { (cívica) }\end{array}$ & $\begin{array}{l}\text { 2. Dominio } \\
\text { de segundo } \\
\text { idioma } \\
\text { (inglés) }\end{array}$ & $\begin{array}{l}\text { 3. Dominio } \\
\text { de } \\
\text { estrategias y } \\
\text { habilidades } \\
\text { de docencia }\end{array}$ & $\begin{array}{l}\text { 4. Desarrollo } \\
\text { de } \\
\text { investigación } \\
\text { científica }\end{array}$ & $\begin{array}{l}\text { 5. Desarrollo } \\
\text { de la } \\
\text { comunidad/ } \\
\text { sociedad }\end{array}$ & $\begin{array}{l}\text { 6. Dominio } \\
\text { de } \\
\text { aprendizajes } \\
\text { en } \\
\text { plataformas } \\
\text { virtuales }\end{array}$ & $\begin{array}{c}7 . \\
\text { Análisis } \\
\text { crítico }\end{array}$ & $\begin{array}{l}\text { 8. Escritura } \\
\text { gramatical } \\
\text { clara y fluida }\end{array}$ \\
\hline Mucho & 70.1 & 82.6 & 80.2 & 90.1 & 81.0 & 79.2 & 93.0 & 87.8 \\
\hline Poco & 24.7 & 12.5 & 16.1 & 7.8 & 15.4 & 17.2 & 4.7 & 8.1 \\
\hline Nada & 1.6 & 1.6 & .5 & .3 & .5 & .8 & 2.3 & .3 \\
\hline $\mathrm{n} / \mathrm{c}$ & 3.7 & 3.4 & 3.2 & 1.8 & 3.1 & 2.9 & & 3.9 \\
\hline Total & 100.0 & 100.0 & 100.0 & 100.0 & 100.0 & 100.0 & 100.0 & 100.0 \\
\hline
\end{tabular}


El nivel de dominio que tienen sobre los mismos elementos se dispersa del de $28 \%$ a $77 \%$, siendo el puntaje más bajo para el dominio del idioma inglés (28.6\%) y conocimiento y promoción de cultura cívica (38.5\%). Para las categorías de investigación (45.1\%) y proyección social (46.6\%) también resulta muy baja en relación al peso que estas dos funciones tienen en la educación superior. El dominio de estrategias y habilidades de docencia con $68.8 \%$. Ver tabla 2.

\section{Tabla 2. Qué nivel de dominio tiene de los siguientes elementos (competencias y habilidades)}

\begin{tabular}{|c|c|c|c|c|c|c|c|c|}
\hline $\begin{array}{l}\text { Nivel } \\
\text { de } \\
\text { dominio } \\
/ \%\end{array}$ & $\begin{array}{l}\text { 1. Conocer } \\
\text { y promover } \\
\text { cultura } \\
\text { política } \\
\text { (cívica) }\end{array}$ & $\begin{array}{l}\text { 2. Dominio } \\
\text { de segundo } \\
\text { idioma } \\
\text { (inglés) }\end{array}$ & $\begin{array}{l}\text { 3. Dominio de } \\
\text { estrategias y } \\
\text { habilidades de } \\
\text { docencia }\end{array}$ & $\begin{array}{l}\text { 4. Desarrollo de } \\
\text { investigación } \\
\text { científica }\end{array}$ & $\begin{array}{l}\text { 5. Desarrollo } \\
\text { de la } \\
\text { comunidad/ } \\
\text { sociedad }\end{array}$ & $\begin{array}{l}\text { 6. Dominio de } \\
\text { aprendizajes } \\
\text { en plataformas } \\
\text { virtuales }\end{array}$ & $\begin{array}{l}7 . \\
\text { Análisis } \\
\text { crítico }\end{array}$ & $\begin{array}{l}8 . \\
\text { Escritura } \\
\text { gramatical } \\
\text { clara y } \\
\text { fluida }\end{array}$ \\
\hline Mucho & 38.5 & 28.6 & 68.8 & 45.1 & 46.6 & 50.3 & 77.9 & 68.2 \\
\hline Poco & 50.8 & 51.6 & 29.2 & 44.8 & 43.5 & 40.4 & 19.3 & 26.8 \\
\hline Nada & 7.6 & 18.0 & .8 & 9.1 & 7.6 & 7.3 & .5 & .8 \\
\hline $\mathrm{n} / \mathrm{c}$ & 3.1 & 1.8 & 1.3 & 1.0 & 2.4 & 2.1 & 2.4 & 4.2 \\
\hline Total & 100.0 & 100.0 & 100.0 & 100.0 & 100.0 & 100.0 & 100.0 & 100.0 \\
\hline
\end{tabular}

Existe una cantidad de docentes que consideran poca importancia y poco dominio de esos elementos, quienes claramente anotan que para ellos es poco o nada importante. Esos porcentajes andan alrededor del 20\%. En cuanto al nivel de dominio, los porcentajes de poco o nada alcanzan hasta el 50\%. De manera que estos datos muestran la necesidad de fortalecer estas competencias entre los docentes de universidades privadas.

Para establecer diferencias más claras en el nivel de mucha importancia y mucho dominio de esos elementos, se presenta la siguiente tabla 3 .

\section{Tabla 3. Diferencia de importancia y dominio de elementos clave para la educación}

\begin{tabular}{|c|c|c|c|c|c|c|c|c|}
\hline Elementos & $\begin{array}{l}\text { 1. Conocer } \\
\text { y promover } \\
\text { cultura } \\
\text { política } \\
\text { (ć́vica) }\end{array}$ & $\begin{array}{l}\text { 2. Dominio } \\
\text { de } \\
\text { segundo } \\
\text { idioma } \\
\text { (inglés) }\end{array}$ & $\begin{array}{l}\text { 3. Dominio } \\
\text { estrategias y } \\
\text { habilidades } \\
\text { de docencia }\end{array}$ & $\begin{array}{l}\text { 4. Desarrollo } \\
\text { de } \\
\text { investigación } \\
\text { científica }\end{array}$ & $\begin{array}{l}\text { 5. Desarrollo } \\
\text { de la } \\
\text { comunidad/ } \\
\text { sociedad }\end{array}$ & $\begin{array}{l}\text { 6. Dominio de } \\
\text { aprendizajes } \\
\text { en plataformas } \\
\text { virtuales }\end{array}$ & $\begin{array}{l}7 . \\
\text { Análisis } \\
\text { crítico }\end{array}$ & $\begin{array}{l}8 . \\
\text { Escritura } \\
\text { gramatical } \\
\text { clara y } \\
\text { fluida }\end{array}$ \\
\hline $\begin{array}{l}\text { Mucha } \\
\text { importancia }\end{array}$ & 70.1 & 82.6 & 80.2 & 90.1 & 81.0 & 79.2 & 93.0 & 87.8 \\
\hline $\begin{array}{l}\text { Mucho } \\
\text { dominio }\end{array}$ & 38.5 & 28.6 & 68.8 & 45.1 & 46.6 & 50.3 & 77.9 & 68.2 \\
\hline Diferencia & $31.6 \%$ & $54 \%$ & $11.4 \%$ & $45 \%$ & $34.4 \%$ & $28.9 \%$ & $15.1 \%$ & $19.6 \%$ \\
\hline
\end{tabular}

Al hacer un balance de niveles de importancia y de dominio solo para quienes contestaron en la categoría de mucho, se evidencia que los niveles de dominio están por debajo de los niveles de importancia. Las mayores diferencias, en promedio de $45 \%$, se ubican para el uso de idioma inglés, investigación y desarrollo de la comunidad/sociedad (equivalente de proyección social), también las categorías de uso de plataformas virtuales y cultura cívica aparecen con promedio de diferencia 
de $30 \%$. Al menos, la mitad de los docentes necesitan fortalecer los elementos analizados para sumar a las competencias necesarias para formar profesionales y contribuir de mejor manera al contexto social de las universidades.

La escritura gramatical clara y fluida facilita la comprensión de la lectura y la claridad para comunicarse. En sintonía con Garrido (2014), cuando se refiere a la escritura y lectura, enfatiza que la lectura posibilita la capacidad de entender, dar y pedir explicaciones, la continuidad del aprendizaje, permite la multiplicación de experiencias, favorece la capacidad de ser autodidactas, cada quien sabe hasta dónde lee, es una manera de adiestrarse en el arte de pensar. Leer y escribir son dos condiciones obligatorias para los académicos, y como base para el desarrollo de competencias, posibilita las estrategias de aprendizaje, tanto como el análisis crítico y el razonamiento lógico.

Al menos el 20\% de los docentes consideran como de poca importancia y poco dominio de los elementos planteados como esenciales en docencia universitaria, quienes claramente anotan que para ellos es poco o nada importante.

\section{CONCLUSIONES Y RECOMENDACIONES}

Los docentes universitarios muestran claridad de la importancia de tener altas competencias y actualización constante, pero a la vez consideran la organización institucional: así son importantes también los recursos y condiciones para desarrollar la docencia. Los niveles de dominio de elementos claves de docencia están por debajo de los niveles de importancia.

Los docentes evidencian dominio (68.8\%) de estrategias y habilidades de docencia universitaria. Este dato se muestra más bajo que lo reportado en el indicador de formación en docencia, que correspondió a $76.6 \%$. Esto puede significar que algunos docentes, a pesar de tener formación en docencia, no dominan estrategias y habilidades en educación terciaria.

Lo anterior significa que aproximadamente la mitad de los docentes de universidades privadas estarían necesitando mejorar los niveles de comprensión y dominio de los elementos analizados, ellos son a quienes deben estar dirigidos los procesos de actualización en esas áreas.

Al considerar que todo conocimiento no es indefinido, que se vuelve obsoleto con rapidez y que una nueva tecnología cambia la realidad, que todo conocimiento es expansivo y relativo a lo local, nacional o internacional y que solo es permanente la capacidad, que la base genérica es permanente e incluye la constante disposición de "aprender a aprender" que debe caracterizar a los académicos, se presentan algunas propuestas de mejora.

\section{Recomendaciones para las 22 Univer- sidades privadas participantes:}

Para las universidades es determinante crear y actualizar un inventario de fortalezasy debilidades delas competencias requeridas para sus docentes, que puede ser en periodo anual y bianual. A partir del inventario actualizado, se recomienda crear e intercambiar programas para la formación y actualización en el tema del "Fortalecimiento de la docencia superior" con otras IES, incorporar a los docentes hora clase y tiempo parcial en programas de formación y actualización profesional, anualmente; considerar que seis de cada diez docentes son hora clase y que el dato tiende a un leve aumento, pues hasta hoy solo se ha priorizado a los docentes tiempo completo que muestran mayor formación de posgrado, que el resto. 
Las IES deben mantener un fuerte equilibrio entre el fortalecimiento del docente, la organización institucional, condiciones y recursos disponibles; para ello se tendrá que planificar las mejoras estratégicas a plazos cortos, medianos y largos. En cuanto a condiciones, las IES deben revisar e incrementar salarios, prestaciones laborales, formación continua del personal docente. Además propiciar condiciones de convivencia armónica que a la vez fortalece valores, muy necesarios para ser exitosos como personas y aprender mejor; la convivencia no se fortalece teóricamente, tanto como en la práctica; esta premisa da espacio para innovaciones didácticas (investigación, aprendizaje basado en proyectos, enseñanza basada en problemas, casos, simulaciones, propiciar el trabajo colaborativo, entre otras).

Potenciar sistemáticamente innovaciones metodológicas en los planes curriculares, como el desarrollo de seminarios al aire libre, visitas a comunidades, instituciones y prácticas de autocuidado dentro de los objetivos del programa de estudios; esto abonará directamente al fortalecimiento de competencias genéricas como base firme para la actualización constante de cualquier especialidad, además de incrementar el bienestar que propicia el buen desempeño.

Las IES, tanto como el MINED, deberían revalorar la incorporación de más docentes de tiempo parcial en las universidades, que por lo general son profesionales que comparten la docencia con la práctica de su profesión. Con un potencial valioso de experiencia, actualmente son menos del $13 \%$, con tendencia de reducción en $2 \%$ por quinquenio.

El objetivo de la actualización permanente es incrementar los niveles de dominio de elementos esenciales, como competencias de base para favorecer un excelente desempeño en docencia universitaria.

\section{ANEXO}

\section{Nombre del programa: "Fortalecimiento de la docencia superior"}

\section{Objetivos específicos y estrategias}

Objetivo específico 1 (OE 1). Aumentar el nivel de competencias en los siguientes elementos (competencias básicas) del desempeño docente por medio de cursos con modalidad de taller.

Temática: Dominio de elementos esenciales de docencia universitaria:

1. Conocer y promover cultura política -cívica (convivencia, cultura de paz, derechos humanos, entre otros).

2. Dominio de segundo idioma (inglés, al menos, en lectura y escritura científica).

3. Dominio de estrategias y habilidades de docencia universitaria (paradigmas, metodología, didáctica de educación superior).

4. Desarrollo de investigación científica (metodología, pertinencia y relevancia social).

5. Desarrollo de la comunidad/sociedad (ABP, EBP, casos, visitas de campo, investigación acción, entre otros).

6. Dominio de aprendizajes en plataformas virtuales, utilización bases de datos y recursos virtuales.

7. Análisis crítico (a partir de seminarios de análisis de la realidad y observación de la realidad)

8. Escritura gramatical clara y fluida (Comunicación escrita y verbal, como base de la comunicación). 
9. Otras que sean de particular importancia en las IES.

Estrategias del objetivo específico 1 (EOE 1)

- Focalizar las instituciones especializadas en cada una de las competencias que se quieren fortalecer y proponer convenios de intercambios de formación en cada una de las áreas señaladas; de manera que se establezca como una especie de canje para que las IES se apoyen financieramente por mutuo acuerdo, sin necesidad de hacer gastos de inscripción.

- Realizar encuentros o congresos en cada una de las áreas señaladas para evaluar el nivel de avance de la mejoras de competencias docentes.

- Desarrollar encuentros o congresos de adecuación curricular, didáctica de educación superior, evaluación, de investigación y de proyección social entre IES y promover la participación de los docentes para evaluar los avances de las competencias requeridas.

- Desarrollar conversatorios docentes para el planteamiento y discusión de casos que impliquen la búsqueda de soluciones conjuntas en el PEA, como el caso de reprobaciones, materias de segunda y tercera matrícula, avance en el logro de perfil de graduados, adecuaciones y flexibilización curricular, etc.

- Aprovechando el talento que hay en las IES. Poner a concurso (consultoría) las actualizaciones de planes y programas de estudio para docentes que tengan el más alto nivel de formación académica y la experiencia requerida en docencia y currículo.

Objetivo específico 2 (OE 2). Aumentar el nivel de formación en posgrado (maestría y doctorado) de los docentes universitarios.
Estrategias de OE 2 (EOE 2)

- Aprovechar los programas de formación de posgrado y cursos especializados que se ofrecen entre las IES, becas completas o parciales por parte de cooperaciones internacionales y apoyar económicamente a quienes obtienen becas parciales.

Recomendaciones para instancias regulatorias de calidad universitaria

Objetivo específico 3 (OE 3). Incorporar indicadores de seguimiento a la calidad de la docencia universitaria.

El MINED y CdA podrían incorporar nuevos indicadores al sistema de calificación y Acreditación, para dar seguimiento al desarrollo de la calidad docente: tanto el MINED, por medio del Sistema de Evaluación, como las agencias de seguimiento a la calidad, como la Comisión de Acreditación de la Educación Superior (CdA). De ser posible, a nivel Centroamericano, por medio del Consejo Centroamericano de Acreditación (CCA) y Sistema Centroamericano de Evaluación y Acreditación de la Educación Superior (SICEVAES-CSUCA). Se deben incorporar nuevos indicadores que muestren la evolución del incremento de competencias docentes, con mediciones que pueden ser anuales. Los indicadores podrían ser: 1- Cantidad de programas de formación que intercambia la institución con las IES nacionales y centroamericanas cada año por medio de acuerdos firmados. 2Cantidad de docentes que participaron en los programas de intercambio con otras IES cada año. 3- Cantidad y nombres de las IES en programas de intercambio. 4- Cantidad de docentes participantes en encuentros de docencia universitaria cada año. 5- Cantidad de docentes que participaron en congresos científicos 
por año. 6- Cantidad de docentes que ingresaron y graduaron de programas de Maestría y Doctorados por año. 7- Cantidad de Planes y Programas curriculares con innovaciones metodológicas y didácticas en modalidad flexible, adecuadas a la enseñanza, actualizados anualmente. 8Porcentaje de cumplimiento del perfil del egresado, entre otros.

Objetivo específico 4 (OE 4). Certificar y Recertificar las competencias académicas de los docentes.

Tanto el MINED, IES y las agencias de calidad deben evaluar a corto y mediano plazo la obligatoriedad de la certificación y recertificación de competencias académicas de docencia universitaria, crear los indicadores de seguimiento. De igual manera podría ser para el resto de profesiones universitarias.

Sin la pretensión de agotar el problema analizado, estas recomendaciones buscan contribuir a lograr mejoras significativas a nivel de docencia universitaria, incluso mejorar el trabajo colaborativo entre las IES salvadoreñas; es una iniciativa que se puede incrementar a nivel centroamericano y regional. Más allá de los esfuerzos que algunas universidades ya tienen, como intercambios a nivel internacional, la iniciativa propuesta tiene el potencial de incluir a la mayor cantidad de docentes en la formación continua y sistematizada, donde se fortalecerán las competencias genéricas a nivel local, con base en la realidad y necesidades comunes de cada institución, y a la vez permite aprovechar el potencial de los profesionales expertos y facilitadores locales.

\section{FUENTES CONSULTADAS}

Álvarez, Margarita María (2011). "Perfil del docente en el enfoque basado en competencias". Revista Electrónica Educare, vol. XV, núm. 1, enero-junio, pp.
99-107. Universidad Nacional Heredia, Costa Rica. Recuperado el 1 de marzo de 2016 de http://www. redalyc.org/articulo.oa?id=194118804008

Beneitone, Esquentini y col. (2007). Proyecto Tuning América Latina 2004-2007. Informe final. Reflexiones y perspectivas de la educación superior en América Latina. Universidad de Deusto, Bilbao España.

Diaz Barriga, Ángel (2005). “El profesor de educación superior frente a las demandas de los nuevos debates educativos". Perfiles educativos, 27 (108), 9-30. Recuperado el 06 de mayo de 2016. http://www.scielo.org.mx/scielo.php?script=sci_ arttext\&pid=S0185-6982005000100002\&lng=es\&t $\operatorname{lng}=\mathrm{es}$.

Garrido, Felipe (2014). "Leer y escribir para ingresar a la educación superior". Revista de la educación superior, 43(172), 145-150. Recuperado en 01 de marzo de 2016. Disponible en http://www.scielo. org.mx/scielo.php?script=sci_arttext \&pid=S018527602014000400008\&lng=es\&tlng=es

Instituto Internacional de la UNESCO para la Educación Superior en América Latina y el Caribe IESALC-UNESCO (1998). Conferencia Mundial de Educación Superior. La educación superior en el siglo XXI. Visión y acción. Paris. CMES, (art. 6), consultado en: http://www.unesco.org/education/educprog/ wche/declaration_spa.htm

Instituto Internacional de la UNESCO para la Educación Superior en América Latina y el Caribe IESALC-UNESCO (2009). Conferencia Mundial de Educación Superior. Las Nuevas Dinámicas de la Educación Superior y de la Investigación para el Cambio Social y el Desarrollo, Paris, 5-8 de julio de 2009. Comunicado, pág. 5. http://www.unesco.org/ education/WCHE2009/comunicado_es.pdf

Instituto Internacional de la UNESCO para la Educación Superior en América Latina y el Caribe, IESALC-UNESCO (2008). Conferencia Regional de Educación Superior (CRES) Cartagena de Indias, Colombia, 4 al 6 de junio de 2008 (IESALC).

Jaques Delors (1996). La educación encierra un tesoro. Informe a la UNESCO de la Comisión Internacional sobre la Educación para el Siglo XXI.

León Urquijo, Ana; Patricia, Risco del Valle, Eduardo, \& Alarcón Salvo, Cristina (2014). "Estrategias de aprendizaje en educación superior en un modelo curricular por competencias". Revista de la educación superior, 43(172), 123-144. Recuperado en 23 de febrero 2016. Disponible en http://www.scielo. org.mx/scielo.php?script=sci_arttext \&pid=S0185$27602014000400007 \& \operatorname{lng}=$ es\&tlng=es 
López, David Ernesto (2012). De la filosofía a la Educación. Fundamentos de una filosofía de la Universidad. Primera Edición. pags. 217-219, 231, 487-5013. Centro de Investigaciones en Ciencias y Humanidades. Universidad Dr. José Matías Delgado.

Ministerio de Educación (2015). Resultados de la Información Estadística de Instituciones de Educación Superior 2014, El Salvador. Págs. 92-98.

Rama, Claudio (2015). Los nuevos modelos universitarios en América Latina. Editorial Univer- sidad Evangélica de El Salvador. San Salvador, El Salvador, C.A (pág.s 50-58).

Rama, Claudio (2016). Mutaciones universitarias latinoamericanas, cambios en las dinámicas educativas, mercados laborales y lógicas económicas. Editorial Universidad Don Bosco. San Salvador, El Salvador, C.A (pags. 25-29, 85-99, 170-175).

Weber, Max (1919). El Político y el científico. La red de Jonás, México, 1979, pag. 71. Original en alemán de Wissendschaftals Beruf Heildenberg. 